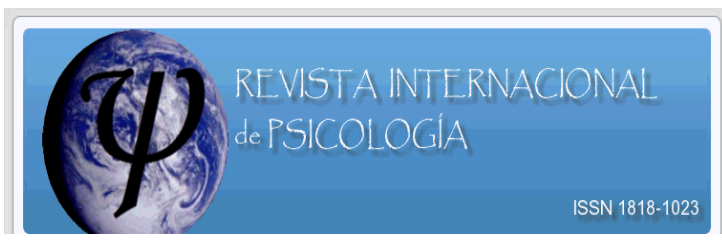

\title{
EL USO DE LAS REDES SEMÁNTICAS NATURALES EN LAS REPRESENTACIONES SOCIALES DE LA RESPONSABILIDAD
}

\author{
Lic. Humberto Emilio Aguilera Arévalo ${ }^{1}$
}

\section{RESUMEN}

El estudio de las representaciones sociales de la responsabilidad es un constructo fundamental de las sociedades democráticas actuales. Las aproximaciones a través de diversas técnicas empíricas como las redes semánticas naturales permite mejorar de manera significativa las técnicas de corte asociacionista que tradicionalmente se han utilizado para acercarse a objeto de estudio. El presente trabajo estudia las redes semánticas naturales de seis palabras estímulo con respecto a la responsabilidad e irresponsabilidad a nivel individual, endogrupal y exogrupal de una muestra de estudiantes universitarios guatemaltecos.

Palabras clave: redes semánticas naturales, responsabilidad, representaciones sociales

\begin{abstract}
The study of social representations of responsibility is a fundamental construct of the present democratic societies. Different empirical techniques such as natural semantic networks can significantly improve the approach to the object of study than the traditional associationist techniques. The present study examines natural semantic networks of six stimulus words with respect to responsibility and irresponsibility at the individual, in group and out group level in a sample of Guatemalan students.
\end{abstract}

Key words: natural semantic networks, responsibility, social representations

\section{INTRODUCCIÓN}

El estudio de las representaciones sociales de la responsabilidad se vuelve fundamental ante el desafío actual de occidente por comprender mejor este constructo, debido principalmente por el aumento de la violencia, conductas negligentes y reclamos de más derechos individuales en las nuevas sociedades democráticas. Su naturaleza básica de "ser capaz de responder" establece una conexión entre el individuo y la sociedad en donde implica el contexto relacional entre lo que alguien demanda y la respuesta que el otro da.

El término responsabilidad, como McKeon (1957) señaló, empezó a aparecer en Europa en el siglo XVII y XVIII y su análisis llevó inevitablemente a las discusiones sobre el libre albedrío versus determinismo. De hecho, fue durante la Ilustración que se formó la idea del hombre como ser independiente de los fenómenos sobrenaturales y, al mismo tiempo profundamente arraigado de la historia y de la naturaleza. La responsabilidad en este sentido trata acerca de vivir interrelacionado con el contexto histórico y cultural (Schulz, 1988).

Tomando un enfoque global del fenómeno, diversas culturas miran a la responsabilidad de diferentes maneras en cómo ser responsable. Winston (2001) señaló

\footnotetext{
${ }^{1}$ Universidad Masaryk Brno, República Checa. Correo electrónico: aguilera99@ yahoo.com
} 
que el concepto occidental moderno de la responsabilidad nunca ha estado muy alejado de su raíz etimológica, que es la capacidad de responder. Si se va a otras culturas, hay diferentes nociones de responsabilidad, cada uno con su propia historia. Cada lengua, inevitablemente, sella el término responsabilidad con su propio ingenio y limitaciones. Para los antiguos griegos y japoneses de hoy, la palabra responsabilidad tiene fuertes connotaciones de culpabilidad que para la palabra en español o en inglés. Para los antiguos griegos una persona es responsable (aitios) cuando la culpa (aitia) por algo pesa sobre sus hombros. Por lo tanto Aristóteles no trata la responsabilidad como una virtud. Por otro lado, los japoneses modernos no suelen asumir la responsabilidad (sekinin) para el éxito, sino sólo para el fracaso. En su cultura, piensan acerca de la responsabilidad en situaciones en las que puede considerárseles responsables, es decir, en situaciones que les gustarían evitar. Sin embargo, las personas con un sentido de responsabilidad (sekininkan ga aru hito) son muy apreciadas, aunque sólo sea porque están seguros de hacer frente a situaciones embarazosas de una manera honesta y directa. Villegas-Reimers (1988) encontró sinónimos de responsabilidad, como la causalidad, la culpa, el juicio moral, el control, la disciplina personal en adolescentes venezolanos en una investigación que hizo desde la psicología social. En la transición política también existen controversias acerca de la noción de responsabilidad en ciertas culturas. Un psicólogo ruso, Andrei Brushlinski, (1994) investigó sobre la controversia en Rusia durante la transición a la democracia después de la caída del comunismo. El notó una tendencia general de que las personas no se sentían responsables por sus propias acciones. Pero en este caso la tendencia a no sentirse responsable, no se debió al énfasis en los derechos individuales. Era algo que se había prorrogado del pasado régimen comunista. Los psicólogos a menudo comentan que el sistema soviético totalitario, que controlaba tanto la vida individual como colectiva, daba a los individuos tan pocas responsabilidades que habían interiorizado una actitud de desesperanza aprendida. Analizando este problema, Brushlinski sostuvo que la década de 1990 fue el momento en que la gente rusa reabrió el debate sobre la moralidad y la responsabilidad haciendo un llamado a la restauración de las conductas sociales basadas en la responsabilidad.

En los estudios transculturales se ha documentado diversas formas en que los individuos conceptualizan el yo. Estos descubrimientos tienen implicaciones importantes para la comprensión de la atribución de responsabilidad y que también plantean importantes desafíos a la teoría. Los psicólogos culturales han encontrado que los individuos de los países occidentales tienden a ver el yo como un ente independiente, mientras que las personas en los países orientales consideran que el yo está estrechamente conectado a los individuos y al contexto en el que viven (Markus y Kitayama, 1991 ; Nisbett, 1998; Triandis, 1989). Shweder y Bourne (1982) les pidieron a estadounidenses indígenas y no indígenas que describieran a sus compañeros. Los investigadores encontraron que los estadounidenses no indígenas utilizan mayoritariamente rasgos atribucionales sin relacionar a sus compañeros a su contexto cuando los describieron, mientras que la mayoría de los estadounidenses indígenas utilizaron descripciones relacionadas al contexto como "él es egoísta solo cuando está en casa".

En las diversas aproximaciones al estudio de la responsabilidad, se encuentran las representaciones sociales que "son construidas sobre esos fenómenos sociales, objeto de preocupación pública, de los que la gente piensa, discute, que causan tensiones y favorecen a la acción" (Markova, 2003, 143). En palabras más sencillas 
representan formas del conocimiento del sentido común, que se construye acerca de los objetos sociales en cuestión. Existen muchas formas de estudiar las representaciones sociales y una de ellas es a través de la técnica de las redes semánticas naturales. Según Figueroa (1976) las define como redes de significados, que son las concepciones que las personas hacen de cualquier objeto de su entorno, mediante el conocimiento de ellas se vuelve factible conocer a través de la multiplicidad de significados, expresados a través del lenguaje cotidiano que tiene todo objeto social.

El modelo de las redes semánticas naturales intenta abordar el estudio del significado, directamente en personas y no a través de modelos de ordenadores.

Además, este modelo intenta dar una explicación del problema que hay acerca de las relaciones que se dan entre los nodos conceptuales que determinan la estructura básica de la red (Figueroa, 1980a), tomando en cuenta que el significado es un componente primordial de la memoria a largo plazo, que implica un proceso de carácter reconstructivo y dinámico que se da a partir del conocimiento y de las relaciones entre conceptos, que se expresan simbólicamente a través del lenguaje.

Figueroa, González y Solís (1981b) demostraron la superioridad de la técnica, que básicamente consiste en pedir a los sujetos que definan la palabra estímulo que se pretende explorar, con un mínimo de cinco palabras sueltas, pudiendo ser nombres, pronombres, sustantivos, adjetivos, verbos, sin usar preposiciones, conjunciones, artículos o cualquier otra partícula gramatical. Posteriormente se les pide a los sujetos que jerarquicen cada una de las palabras definidoras de acuerdo al orden de importancia que cada una de ellas tiene respecto a la palabra estímulo. Para ello, le asignan el número uno a la palabra que mejor define a la palabra estímulo, el número dos a la que le sigue y así sucesivamente hasta terminar de jerarquizar todas las palabras que escribieron.

Con ello, se puede demostrar que a pesar de que la técnica tiene orígenes asociacionistas, los resultados son más que asociaciones libres de conceptos, puesto que al solicitar a los sujetos la jerarquización de cada una de las palabras, hace que se obtenga precisamente una red semántica, entendida como el conjunto de conceptos seleccionados por los procesos de reconstrucción de la memoria, considerando que esta selección, no es una simple asociación, ya que está determinada por las clases y propiedades de los elementos que la integran.

La técnica de redes semánticas, ofrece un medio empírico de acceso a la organización cognitiva del conocimiento. Por tanto, puede proporcionar datos referentes a la organización e interpretación interna de los significantes. También indica como la información, fue percibida individualmente en el curso de la composición del aprendizaje social y provee indicios fundamentales, a cerca de la tendencia a actuar basándose en ese "universo cognitivo". Krech (1975), supone que el ambiente físico y el social aprendido por el individuo facilitan el entendimiento del mundo social y solución de problemas. Por tanto, suponen, que existen problemas sociales, que provocan necesidades comunes a las personas, llevándolas a organizar el conocimiento ganado empíricamente con el fin de actuar, eficazmente, de manera colectiva frente a situaciones específicas.

Según Tulving (1972), la técnica de redes semánticas, se deriva de los estudios de la memoria en largo plazo en el campo de la Psicología cognitiva. De acuerdo con 
Morales (1994) la memoria semántica es construida por representaciones de conceptos y conocimientos generales. La memoria semántica es la memoria necesaria para el uso del lenguaje, organiza el conocimiento que las personas poseen de las palabras y otros símbolos verbales, sus significados y referentes acerca de las relaciones entre ellos y de las reglas, fórmulas y algoritmos para la manipulación de los símbolos, conceptos y relaciones. Grzib y Briales (1996) concluyen que la memoria semántica toma en cuenta la capacidad humana para construir la realidad, en una interpretación interna y es a través de ésta, que se decodifican las experiencias pasadas, predicciones y causalidades, se conectan dentro de combinaciones nuevas.

\section{METODOLOGÍA}

\section{Muestra}

Se trabajó con una muestra no probabilística de cuota compuesta por 2 grupos de 148 sujetos de ambos sexos, de las carreras de ingenierías y humanidades de 2 universidades privadas de Guatemala.

La muestra estuvo compuesta de 296 estudiantes universitarios (Edad Media $=$ 21,7, Desviación Estándar = 5,07), de los cuales el 190 eran mujeres (el 64\%, Edad M = 22,14, Desviación Estándar = 5,95) y 106 eran hombres (el 36\%, Edad M = 20,91, Desviación Estándar = 2,78)

E1 94,3\% de los estudiantes eran solteros y el 96,6\% originarios de la ciudad capital. El $80.5 \%$ eran exclusivamente estudiantes, es decir que no trabajaban, y el $81,7 \%$ vivían con sus padres. El 81,5\% de sus padres eran casados. El 72,4\% de sus padres eran universitarios, sin embargo un $22,9 \%$ habían estudiado bachillerato. Un $50,5 \%$ de sus madres eran universitarios, sin embargo un 39,9\% habían estudiado bachillerato.

Un $12.4 \%$ de los padres de los sujetos eran gerentes y un $12 \%$ eran ingenieros. Los estudiantes de ingeniería tenían más padres gerentes que los de humanidades por un $4.4 \%$

Un $37.3 \%$ de las madres de los sujetos eran amas de casa y un $6.7 \%$ eran maestras. Los estudiantes de humanidades tenían más madres amas de casa que los de humanidades por un $2.8 \%$

El 70.6\% de los sujetos conocían a un europeo. De ese $70.6 \%$ de los que conocían a un europeo, un $23.4 \%$ conocían a un español, un $15.9 \%$ a un alemán y un $14.1 \%$ a un francés

Los estudiantes de ingeniería conocían por un $2.2 \%$ más a españoles y por un $2.8 \%$ más a un francés. 


\section{Instrumentos}

Se aplicó el siguiente cuestionario:

RedessemánticasnaturalesdeValdezMedina: Para las palabras estímulo Yo responsable, Yo irresponsable, Guatemalteco responsable, Guatemalteco irresponsable, Europeo responsable, Europeo irresponsable

\section{Procedimiento}

En la aplicación de las redes semánticas naturales se les pidió a los sujetos que definieran con un mínimo de cinco palabras sueltas que podían ser: verbos, adverbios, sustantivos, adjetivos, etc., sin usar partículas gramaticales como preposiciones o artículos, a cada una de las palabras estímulo (Yo responsable, Yo irresponsable, Guatemalteco responsable, Guatemalteco irresponsable, Europeo responsable, Europeo irresponsable)

Posteriormente, se les pidió que jerarquizaran cada una de las palabras que dieron como definidoras, asignándole el número 1 a la palabra que consideraran que estuviera más cerca, más relacionada o que mejor definiera a la palabra estímulo, el número 2 a la que le sigue en relación, el 3 a la siguiente y así sucesivamente hasta terminar de jerarquizar todas y cada una de las palabras definidoras generadas por los sujetos.

Los cuestionarios fueron distribuidos en 2 universidades privadas ya que se asumió que los estudiantes habían tenido un mayor contacto con alguna persona europea que si se tratara de la universidad nacional. La participación fue voluntaria y la prueba se llenó de manera individual. Los datos fueron recolectados entre febrero y mayo del 2010.

\section{Análisis}

Se realizó el análisis respectivo en las redes semánticas naturales, obteniendo el grupo SAM, es decir el grupo de 15 palabras definidoras con mayor peso semántico, de cada palabra estímulo. Además se realizó el conteo de frecuencias del grupo SAM respectivo.

\section{RESULTADOS}

A partir de los datos obtenidos por las redes semánticas naturales se obtuvo que para la palabra estímulo Yo responsable, la riqueza semántica fue de 500 palabras y se encontró que fue definido por los sujetos como puntual, cumplido, ordenado, trabajador, organizado, honesto, dedicado, respetuoso, estudioso, trabajo, tareas, estudio, comprometido, atento y disciplina. Hubo diferencias en la jerarquía de frecuencias de las palabras definidoras con la de la red semántica natural en las palabras honesto, trabajador, dedicado, organizado, atento, estudio, comprometido y consciente. (ver tabla 1). 


\begin{tabular}{|l|r|r|l|r|}
\hline \multicolumn{5}{|c|}{ Conjunto SAM para YO RESP 1 } \\
CONJUNTO & $\begin{array}{l}\text { VALO } \\
\text { R M }\end{array}$ & $\begin{array}{l}\text { VALO } \\
\text { R FMG }\end{array}$ & & $\begin{array}{l}\text { FRECUENCIA } \\
\text { SAM }\end{array}$ \\
\hline puntual & 1088 & $100 \%$ & puntual & 153 \\
\hline cumplido & 715 & $66 \%$ & cumplido & 93 \\
\hline ordenado & 581 & $53 \%$ & ordenado & 93 \\
\hline trabajador & 562 & $52 \%$ & honesto & 79 \\
\hline organizado & 505 & $46 \%$ & trabajador & 76 \\
\hline honesto & 504 & $46 \%$ & dedicado & 64 \\
\hline dedicado & 471 & $43 \%$ & organizado & 63 \\
\hline respetuoso & 330 & $30 \%$ & respetuoso & 54 \\
\hline estudioso & 322 & $30 \%$ & estudioso & 52 \\
\hline trabajo & 310 & $28 \%$ & trabajo & 50 \\
\hline tareas & 291 & $27 \%$ & tareas & 48 \\
\hline estudio & 266 & $24 \%$ & atento & 44 \\
\hline comprometido & 247 & $23 \%$ & estudio & 39 \\
\hline & & & comprometid & 30 \\
\hline atento & 211 & $19 \%$ & o & 28 \\
\hline disciplina & 194 & $18 \%$ & consciente & \\
\hline $\boldsymbol{j}=\mathbf{5 0 0}$ & & & & \\
\hline
\end{tabular}

A partir de los datos obtenidos por las redes semánticas naturales se obtuvo que para la palabra estímulo Guatemalteco responsable, la riqueza semántica fue de 552 palabras y se encontró que fue definido por los sujetos como trabajador, puntual, cumplido, respetuoso, honesto, trabajo, ordenado, organizado, honrado, dedicado, consciente, patriota, solidario, amable y familia. Hubo diferencias en la jerarquía de frecuencias de las palabras definidoras con la de la red semántica natural en las palabras ordenado, trabajo, amable, consciente, solidario, honrado, dedicado, atento y limpio. (ver tabla 2).

\begin{tabular}{|c|c|c|c|c|}
\hline \multicolumn{5}{|c|}{$\begin{array}{c}\text { Tabla } 2 \\
\text { Conjunto SAM para GUATEMALTECO RESPONSABLE y } \\
\text { frecuencias }\end{array}$} \\
\hline $\begin{array}{l}\text { CONJUNTO } \\
\text { SAM }\end{array}$ & $\begin{array}{l}\text { VALO } \\
R M\end{array}$ & $\begin{array}{l}\text { VALO } \\
\text { R FMG }\end{array}$ & & $\begin{array}{l}\text { FRECUENCIA } \\
S\end{array}$ \\
\hline trabajador & 1047 & $100 \%$ & trabajador & 120 \\
\hline puntual & 651 & $62 \%$ & puntual & 93 \\
\hline cumplido & 486 & $46 \%$ & cumplido & 65 \\
\hline respetuoso & 413 & $39 \%$ & $\begin{array}{l}\text { respetuos } \\
0 \\
\end{array}$ & 64 \\
\hline honesto & 395 & $38 \%$ & honesto & 58 \\
\hline trabajo & 338 & $32 \%$ & \begin{tabular}{|l|} 
ordenado \\
\end{tabular} & 49 \\
\hline ordenado & 322 & $31 \%$ & trabajo & 43 \\
\hline organizado & 294 & $28 \%$ & $\begin{array}{l}\text { organizad } \\
\text { o }\end{array}$ & 42 \\
\hline honrado & 203 & $19 \%$ & amable & 35 \\
\hline dedicado & 187 & $18 \%$ & consciente & 29 \\
\hline consciente & 186 & $18 \%$ & solidario & 29 \\
\hline patriota & 175 & $17 \%$ & honrado & 28 \\
\hline solidario & 172 & $16 \%$ & dedicado & 28 \\
\hline amable & 158 & $15 \%$ & atento & 27 \\
\hline familia & 151 & $14 \%$ & limpio & 26 \\
\hline$j=552$ & & & & \\
\hline
\end{tabular}


A partir de los datos obtenidos por las redes semánticas naturales se obtuvo que para la palabra estímulo Europeo responsable, la riqueza semántica fue de 503 palabras y se encontró que fue definido por los sujetos como puntual, trabajador, respetuoso, organizado, trabajo, cumplido, educado, ordenado, honesto, limpio, consciente, estudioso, exitoso, amable, cultura. Hubo diferencias en la jerarquía de frecuencias de las palabras definidoras con la de la red semántica natural en las palabras ordenado, honesto, cumplido, limpio, educado, amable, estudioso, cultura e inteligente. (ver tabla $3)$.

\begin{tabular}{|c|c|c|c|c|}
\hline \multicolumn{5}{|c|}{$\begin{array}{c}\text { Tabla } 3 \\
\text { Conjunto SAM para EUROPEO RESPONSABLE y frecuencias }\end{array}$} \\
\hline $\begin{array}{l}\text { CONJUNTO } \\
\text { SAM }\end{array}$ & $\begin{array}{l}\text { VALO } \\
R M \\
M\end{array}$ & $\begin{array}{l}\text { VALO } \\
\text { R FMG }\end{array}$ & & $\begin{array}{l}\text { FRECUENCIA } \\
S\end{array}$ \\
\hline puntual & 755 & $100 \%$ & puntual & 98 \\
\hline trabajador & 650 & $86 \%$ & trabajador & 83 \\
\hline respetuoso & 338 & $45 \%$ & $\begin{array}{l}\text { respetuos } \\
0\end{array}$ & 52 \\
\hline organizado & 281 & $37 \%$ & $\begin{array}{l}\text { organizad } \\
0\end{array}$ & 39 \\
\hline trabajo & 277 & $37 \%$ & trabajo & 38 \\
\hline cumplido & 249 & $33 \%$ & ordenado & 36 \\
\hline educado & 245 & $32 \%$ & honesto & 36 \\
\hline ordenado & 241 & $32 \%$ & cumplido & 34 \\
\hline honesto & 238 & $32 \%$ & limpio & 33 \\
\hline limpio & 170 & $23 \%$ & educado & 31 \\
\hline consciente & 150 & $20 \%$ & consciente & 21 \\
\hline estudioso & 138 & $18 \%$ & amable & 20 \\
\hline exitoso & 121 & $16 \%$ & estudioso & 19 \\
\hline amable & 121 & $16 \%$ & cultura & 19 \\
\hline cultura & 120 & $16 \%$ & inteligente & 19 \\
\hline$j=503$ & & & & \\
\hline
\end{tabular}

A partir de los datos obtenidos por las redes semánticas naturales se obtuvo que para la palabra estímulo Yo irresponsable, la riqueza semántica fue de 591 palabras y se encontró que fue definido por los sujetos como impuntual, desordenado, haragán, desorganizado, incumplido, perezoso, dejado, olvidadizo, irrespetuoso, descuidado, despreocupado, desinteresado, deshonesto, mentiroso y malo. Hubo diferencias en la jerarquía de frecuencias de las palabras definidoras con la de la red semántica natural en las palabras irrespetuoso, dejado, despreocupado, desinteresado, descuidado, malo y mentiroso. (ver tabla 4). 


\begin{tabular}{|l|r|r|l|r|}
\hline \multicolumn{5}{|c|}{ Conjunto SAM para YO IRRESPONSABLE y frecuencias } \\
\hline $\begin{array}{l}\text { CONJUNTO } \\
\text { SAM }\end{array}$ & $\begin{array}{l}\text { VALO } \\
\text { R M }\end{array}$ & $\begin{array}{l}\text { VALO } \\
\text { R FMG }\end{array}$ & & $\begin{array}{l}\text { FRECUENCIA } \\
\text { S }\end{array}$ \\
\hline impuntual & 887 & $100 \%$ & impuntual & 119 \\
\hline desordenado & 732 & $83 \%$ & desordenado & 102 \\
\hline haragán & 597 & $67 \%$ & haragán & 79 \\
\hline desorganizado & 511 & $58 \%$ & desorganizado & 68 \\
\hline incumplido & 455 & $51 \%$ & incumplido & 55 \\
\hline perezoso & 361 & $41 \%$ & perezoso & 48 \\
\hline dejado & 273 & $31 \%$ & irrespetuoso & 40 \\
\hline olvidadizo & 265 & $30 \%$ & olvidadizo & 39 \\
\hline irrespetuoso & 241 & $27 \%$ & dejado & 38 \\
\hline & 234 & $26 \%$ & o spreocupad & 30 \\
\hline descuidado & 228 & $26 \%$ & desinteresado & 30 \\
\hline despreocupado & 218 & $25 \%$ & descuidado & 29 \\
\hline desinteresado & 188 & $21 \%$ & deshonesto & 26 \\
\hline deshonesto & 143 & $16 \%$ & malo & 22 \\
\hline mentiroso & 133 & $15 \%$ & mentiroso & \\
\hline malo & & & & \\
\hline $\boldsymbol{j}=\mathbf{5 9 1}$ & & & & \\
\hline
\end{tabular}

A partir de los datos obtenidos por las redes semánticas naturales se obtuvo que para la palabra estímulo Guatemalteco irresponsable, la riqueza semántica fue de 576 palabras y se encontró que fue definido por los sujetos como impuntual, haragán, desordenado, irrespetuoso, incumplido, perezoso, desorganizado, mentiroso, deshonesto, desinteresado, ladrón, corrupto, dejado, despreocupado, vago. Hubo diferencias en la jerarquía de frecuencias de las palabras definidoras con la de la red semántica natural en las palabras mentiroso, incumplido, perezoso, desorganizado, ladrón, deshonesto, sucio, desinteresado, corrupto y dejado. (ver tabla 5).

\begin{tabular}{|l|r|r|l|r|}
\hline \multicolumn{5}{|c|}{ Conjunto SAM para GUATEMALTECO IRRESPONSABLE y } \\
frecuencias
\end{tabular}


A partir de los datos obtenidos por las redes semánticas naturales se obtuvo que para la palabra estímulo Europeo irresponsable, la riqueza semántica fue de 501 palabras y se encontró que fue definido por los sujetos como impuntual, haragán, drogas, irrespetuoso, desordenado, sucio, desorganizado, vago, incumplido, fiestero, egoísta, desinteresado, dejado, descuidado y perezoso. Hubo diferencias en la jerarquía de frecuencias de las palabras definidoras con la de la red semántica natural en las palabras sucio, desordenado, fiestero, egoísta, desinteresado, perezoso, incumplido y mentiroso. (ver tabla 6).

\begin{tabular}{|c|c|c|c|c|}
\hline \multicolumn{5}{|c|}{$\begin{array}{c}\text { Tabla } 6 \\
\text { Conjunto SAM para EUROPEO IRRESPONSABLE y frecuencias }\end{array}$} \\
\hline $\begin{array}{l}\text { CONJUNTO } \\
\text { SAM }\end{array}$ & $\begin{array}{l}\text { VALO } \\
R M\end{array}$ & $\begin{array}{l}\text { VALO } \\
\text { R FMG }\end{array}$ & & $\begin{array}{l}\text { FRECUENCIA } \\
S\end{array}$ \\
\hline impuntual & 364 & $100 \%$ & impuntual & 50 \\
\hline haragán & 355 & $98 \%$ & haragán & 48 \\
\hline drogas & 231 & $63 \%$ & drogas & 34 \\
\hline irrespetuoso & 229 & $63 \%$ & irrespetuoso & 33 \\
\hline desordenado & 224 & $62 \%$ & sucio & 32 \\
\hline sucio & 210 & $58 \%$ & desordenado & 31 \\
\hline desorganizado & 208 & $57 \%$ & $\begin{array}{l}\text { desorganizad } \\
0\end{array}$ & 27 \\
\hline vago & 187 & $51 \%$ & vago & 26 \\
\hline incumplido & 178 & $49 \%$ & fiestero & 25 \\
\hline fiestero & 172 & $47 \%$ & egoísta & 22 \\
\hline egoísta & 170 & $47 \%$ & desinteresado & 21 \\
\hline desinteresado & 166 & $46 \%$ & perezoso & 21 \\
\hline dejado & 152 & $42 \%$ & incumplido & 20 \\
\hline descuidado & 150 & $41 \%$ & descuidado & 20 \\
\hline perezoso & 144 & $40 \%$ & mentiroso & 20 \\
\hline$j=501$ & & & & \\
\hline
\end{tabular}

\section{DISCUSIÓN}

El uso de dicha esta técnica permite observar como se organiza la red semántica a partir de cada palabra estímulo. De acuerdo a lo que menciona Figueroa, González y Solís (1981b) demuestra la superioridad de la técnica al organizar mejor la información y jerarquizar más que una simple asociación de palabras. Como se puede observar en la tabla 1, respecto a lo que los sujetos definen mejor Yo responsable, las palabras más frecuentes y con mayor jerarquía en el grupo SAM fueron puntual, cumplido y ordenado. Respecto a las palabras honesto, trabajador, dedicado, organizado, atento, estudio, comprometido y consciente, difirieron con respecto a la jerarquía en la red semántica natural, lo cual indica que a pesar de ser más o menos frecuente, no es una simple asociación, ya que está determinada por las clases y propiedades de los elementos que la integran. Por ejemplo, a pesar de que la palabra definidora honesto fue más frecuente que trabajador, los sujetos la consideraron menos cercana a definir Yo responsable que la palabra trabajador, que fue menos frecuente. La palabra consciente entra dentro de las 15 palabras más frecuentes, pero no obtuvo suficiente peso semántico para entrar dentro del grupo SAM. En relación a la palabra definidora a nivel 
endogrupal Guatemalteco responsable, las palabras más frecuentes y con mayor jerarquía en el grupo SAM fueron trabajador, puntual, cumplido, respetuoso y honesto. Respecto a las palabras ordenado, trabajo, amable, consciente, solidario, honrado, dedicado, atento y limpio difirieron con respecto a la jerarquía en la red semántica natural. La palabra definidora amable fue más frecuente pero con menor valor semántico en la jerarquía del grupo SAM. Las palabras definidoras honrado y dedicado fueron menos frecuentes, pero con mayor peso semántico en el conjunto SAM. Las palabras atento y limpio no obtuvieron suficiente peso semántico para entrar dentro del grupo SAM. Con respecto a la palabra definidora a nivel exogrupal Europeo responsable, las palabras más frecuentes y con mayor jerarquía en el grupo SAM fueron puntual, trabajador, respetuoso, organizado y trabajo. Respecto a las palabras ordenado, honesto, cumplido, limpio, educado, amable, estudioso, cultura e inteligente difirieron con respecto a la jerarquía en la red semántica natural. Las palabras cumplido y educado fueron menos frecuentes pero con mayor peso semántico en el conjunto SAM. La palabra inteligente a pesar de ser frecuente no aparece dentro del conjunto SAM. En relación a la palabra definidora Yo irresponsable, las palabras más frecuentes y con mayor jerarquía en el grupo SAM fueron impuntual, desordenado, haragán, desorganizado, incumplido y perezoso. Respecto a las palabras irrespetuoso, dejado, despreocupado, desinteresado, descuidado, malo y mentiroso difirieron con respecto a la jerarquía en la red semántica natural. Las palabras definidoras irrespetuoso, despreocupado y malo fueron más frecuentes pero los sujetos le dieron menor valor semántico en el conjunto SAM. No hubo ninguna palabra frecuente que no apareciera en el grupo SAM. En relación a la palabra definidora a nivel endogrupal Guatemalteco irresponsable, las palabras más frecuentes y con mayor jerarquía en el grupo SAM fueron impuntual, haragán, desordenado e irrespetuoso. Las palabras mentiroso, incumplido, perezoso, desorganizado, ladrón, deshonesto, sucio, desinteresado, corrupto y dejado difirieron con respecto a la jerarquía en la red semántica natural. Las palabras mentiroso y ladrón fueron más frecuentes pero obtuvieron menor peso semántico en el conjunto SAM. La palabra definidora sucio no entró dentro del grupo SAM a pesar de ser tan frecuente como las otras. Con respecto a la palabra definidora a nivel exogrupal Europeo irresponsable, las palabras más frecuentes y con mayor jerarquía en el grupo SAM fueron impuntual, haragán, drogas e irrespetuoso. Las palabras sucio, desordenado, fiestero, egoísta, desinteresado, perezoso, incumplido y mentiroso difirieron con respecto a la jerarquía en la red semántica natural. Las palabras sucio, fiestero, egoísta, desinteresado y perezoso fueron más frecuentes pero obtuvieron menor peso semántico en el conjunto SAM. La palabra mentiroso no aparece en el grupo SAM a pesar de ser tan frecuente como incumplido y descuidado. 


\section{REFERENCIAS}

Brushlinski A (1994) Problems of the psychology of the subject. Rossijskaja Akademia Nauk, Moscow

Figueroa, J. G. (1976) Estudios de Redes Semánticas Naturales y algunos procesos básicos.Universidad Nacional Autónoma de México: UNAM. p. 56.

Figueroa, J. (1980a) Sobre la teoría general de las redes semánticas. Facultad de psicología de la Universidad Nacional Autónoma de México. Trabajo inédito.

Figueroa, J. G. González, E. G. y Solis, V.M. (1981b) “Una aproximación al problema del significado: las redes semánticas”. Revista Latinoamericana de Psicología 13: 447-458.

Grzib, G. y Briales, C. (1996) "Psicología General". Editorial Centro de Estudios Ramon Areces S.A. Madrid. 64 p.

Krech, D., Crutchfied, R.S. y Ballachey, E.L (1975) “El individuo y la sociedad”. D.M. $42 \mathrm{p}$.

Markova, I. (2003). Dialogicality and social representations. Cambridge: Cambridge University Press.

Markus, H., and Kitayama, S. (1991) Culture and the self: Implications for cognition, emotion and motivation, Psychological Review 98: 224-254

McKeon, R. (1957). The development and the Significance of the Concept of Responsibility. Revue Internationale de Philosophie, 39, 3-32.

Nisbett, R.E. (1998) Essence and accident, in J.M. Darley and J.Cooper (eds), Attribution in Social Interaction: The legacy of Ned Jones, pp.171-197, Washington, DC: American Psychological Association. 
Schulz, W. (1988). Le nuove vie della filosofia contemporanea, vol. 5, Responsabilità. Genova: Marietti.

Shweder, R.A., and Bourne, E.J. (1982) Does the concept of the person vary crossculturally? In A.J.Marsella and G.M.White (eds), Cultural Conceptions of Mental Health and Therapy, Dordrech, Holland: D.Riedel

Triandis, H. (1989) The self and social behavior in different cultural contexts, Psychological Review 96:506-520

Tulving, E. y Pearlstone (1972) "Episodic and Semantic Memory", "Organization of Memory”. Academic Press. New York. 56 p.

Villegas-Reimers, "Judgments of Responsibility: Their Relationship with Self and Moral Reasoning in Venezuelan Adolescents,” DAE 49 (1989): 2597A (Harvard Graduate School of Education, 1988).

Winston, Davis. (2001). Taking responsibility. Comparative Perspectives. The University Press of Virginia. 5-10 
Usted es libre para Compartir -copiar y redistribuir el material en cualquier medio o formato - y Adaptar el documen- to -remezclar, transformar y crear a partir del material- para cualquier propósito, incluso comercialmente, siempre que cumpla la condición de:

Atribución: Usted debe reconocer el crédito de una obra de manera adecuada, proporcionar un enlace a la licencia, e in- dicar si se han realizado cambios. Puede hacerlo en cualquier forma razonable, pero no de forma tal que sugiera que tie- ne el apoyo del licenciante o lo recibe por el uso que hace. 